\title{
NIVEL DE CONOCIMIENTOS SOBRE EMERGENCIAS MÉDICAS EN ESTUDIANTES DE MEDICINA DE UNIVERSIDADES PERUANAS
}

\author{
Christian R. Mejia1,2,3,a, Claudia Quezada-Osoria ${ }^{4, b}$, Cinthya Moras-Ventocilla ${ }^{3,5, a}$, \\ Kelly Quinto-Porras ${ }^{3,5, a}$, Carlos Ascencios-Oyarce ${ }^{6, a}$
}

\begin{abstract}
RESUMEN
Objetivos. Evaluar el nivel de conocimientos de los estudiantes de medicina de once universidades peruanas sobre emergencias médicas. Materiales y métodos. Estudio transversal analítico, multicéntrico desarrollado entre 20072008. Se utilizó un cuestionario de respuesta nominal autoaplicado con preguntas socio-educativas y diez de opción múltiple sobre emergencias médicas. Se obtuvo una muestra no probabilística de los matriculados en universidades participantes. Se obtuvieron el chi-cuadrado, los OR crudos y ajustados, con intervalos de confianza al $95 \%$ y análisis multivariado posterior. Resultados. Participaron 2109 estudiantes de medicina, la edad promedio fue 21 años (rango: $15-32)$, el $51 \%$ de género masculino. El $53 \%$ habían realizado un curso previo relacionado con emergencias médicas. El $60,4 \%$ desaprobó el cuestionario, la nota promedio fue 4,95 sobre 10 puntos posibles y 5,9\% obtuvieron de 8-10 puntos. Se encontró una fuerte asociación entre la universidad de procedencia (OR: 0,45, IC95\% 0,38-0,54), la etapa académica (OR: 1,55, IC95\% 1,28-1,87), cuándo recibieron un curso del tema (OR: 0,62, IC95\% 0,50-0,77) y el género (OR: 1,38, IC95\% 1,15-1,65). Conclusiones. El nivel de conocimiento sobre emergencias médicas de los estudiantes de las once universidades evaluadas no es bueno, se sugiere evaluar y mejorar la formación práctica que brindan las universidades en temas de manejo de emergencias médicas.
\end{abstract}

Palabras Clave: Urgencias médicas; Estudiantes de medicina; Primeros auxilios (fuente: DeCS BIREME).

\section{LEVEL OF KNOWLEDGE IN MEDICAL EMERGENCIES AMONG MEDICAL STUDENTS OF PERUVIAN UNIVERSITIES}

\begin{abstract}
Objectives. The aim of this study was to evaluate the knowledge about medical emergencies of medical students from eleven Peruvian universities. Materials and methods. Multicenter, cross-sectional study, conducted between 20072008. We used a nominal response, self-administered questionnaire with socio-educational questions and ten multiple choice questions on medical emergencies. We obtained a nonrandom sample of participants enrolled in universities. We obtained the chi2, crude and adjusted ORs with $95 \%$ confidence intervals and ulterior multivariate analysis. Results. 2,109 medical students participated, the average age was 21 years (range: 15-32), 51\% were males. 53\% had taken a previous course related to medical emergencies. $60.4 \%$ failed the questionnaire, the average score was 4.95 over a maximum of 10 points and $5.9 \%$ obtained between $8-10$ points. We found a strong association between the university of origin (OR: $0.45,95 \% \mathrm{Cl} 0.38$ to 0.54 ), the academic stage (OR: $1.55,95 \% \mathrm{Cl} 1.28$ to 1.87 ), when they received the course about subject (OR: $0.62,95 \% \mathrm{Cl} 0.50-0.77$ ) and gender (OR: $1.38,95 \% \mathrm{Cl} 1.15$ to 1.65). Conclusions. Knowledge of medical emergencies in the students of the eleven evaluated universities is not good, and we suggest the need of evaluating and improving the practical training offered by universities on issues regarding the management of medical emergencies.
\end{abstract}

Key words: Emergencies; Students, Medical; First Aids. (source: MeSH NLM).

\section{INTRODUCCIÓN}

Las emergencias médicas son definidas por la Organización Mundial de la Salud (OMS) como situaciones que tienen consecuencias inmediatas riesgosas para la vida (1), por lo que requieren atención inmediata ${ }^{(2)}$. Estas situaciones pueden ser originadas por fenómenos naturales, actos humanos deliberados, enfermedades o por la combinación de ellos (3). En nuestro medio las emergencias médicas más

\footnotetext{
Asociación Médica de Investigación y Servicios y en Salud. Lima, Perú.

2 Comité del Médico Joven. Colegio Médico del Perú. Lima, Perú.

Sociedad Científica de Estudiantes de Medicina de la Universidad Ricardo Palma. Lima, Perú.

Sociedad Científica de Estudiantes de Medicina de la Universidad Nacional de Piura. Piura, Perú.

5 Facultad de Medicina de la Universidad Ricardo Palma. Lima, Perú.

6 Facultad de Medicina de la Universidad Nacional de Cajamarca. Cajamarca, Perú.

a Médico-cirujano; ${ }^{\mathrm{b}}$ Estudiante de medicina.
} 
frecuentes son causadas por accidentes de tránsito o por eventos cardiovasculares agudos ${ }^{(4)}$. Su incidencia ha incrementado durante los últimos años, por lo que es importante impulsar la mejora en la infraestructura de los servicios de salud y, sobre todo, garantizar que el personal de salud esté adecuadamente capacitado para enfrentar este tipo de eventos ${ }^{(5,6)}$.

Los estudiantes de medicina, potencialmente, pertenecen al equipo del personal de salud y, muchas veces, la población acude a ellos por esta característica ${ }^{(7,8)}$. Sin embargo, la educación para el manejo de emergencias médicas que habitualmente se enseña y practica en la carrera de medicina humana parece ser inadecuada, en especial en la atención primaria ${ }^{{ }^{9-12)}}$. Son pocas las facultades de medicina donde desde los primeros años de formación se imparte una enseñanza adecuada en estos tópicos ${ }^{(13-15)}$, los estudiantes son excluidos durante su formación en el tratamiento primario de situaciones críticas (16-18), a pesar que estas representan una de las circunstancias en donde el alumno muestra los auténticos conocimientos académicos y habilidades médicas ${ }^{(19,20)}$. Los estudiantes deberían recibir una enseñanza adecuada, que asegure el correcto actuar de los futuros médicos frente a estos eventos ${ }^{(21-23)}$.

En nuestro medio se han encontrado poca literatura que aborde este problema ${ }^{(24,25)}$. El objetivo de este estudio fue evaluar el nivel de conocimientos de los estudiantes de medicina de once universidades peruanas sobre emergencias médicas.

\section{MATERIALES Y MÉTODOS}

\section{DISEÑO Y POBLACIÓN DE ESTUDIO}

Se realizó un estudio transversal analítico multicéntrico durante los años 2007-2008. Se incluyeron estudiantes de medicina de primero a quinto año matriculados en el período de estudio en alguna de las once universidades peruanas participantes (Universidad Nacional de San Agustín-Arequipa, Nacional de Cajamarca, Pedro Ruiz Gallo-Chiclayo, San Antonio Abad-Cusco, Nacional del Centro-Huancayo, Nacional de Piura, del AltiplanoPuno y Jorge Basadre Groham-Tacna, y las particulares Universidad Católica Santa María-Arequipa, San Pedro-Ancash y Ricardo Palma-Lima), se excluyeron a los estudiantes de sexto y sétimo año puesto que se encuentran en actividades previas al internado médico o realizando dicho internado. Se obtuvo una muestra no probabilística con un nivel de significancia de $5 \%$, para un estudio de dos colas, basándose en una población 6188 estudiantes matriculados en los cinco primeros años en todas las universidades participantes, usan- do un error de 0,02 (para minimizar las probabilidades de error en la selección de sujetos) y un alfa de 1,96, estimando una proporción de promedio aprobatorio de $50 \%$. Se obtuvo un tamaño muestral mínimo de 1730 , a este resultado se le sumó el $10 \%$ por la tasa de rechazo esperada, obteniendo un tamaño muestral final mínimo de 1903 estudiantes de medicina. Se procuró que la muestra de cada universidad sea proporcional a la totalidad de sus alumnos y a la cantidad de alumnos por año. Se usó el paquete estadístico STATA 11.0 (STATA Corp, Texas, US).

\section{INSTRUMENTOS Y VARIABLES}

Se usó un cuestionario tipo test de potencia que empleó una escala de respuesta nominal, el cual fue elaborado con el objetivo de medir el nivel de conocimiento ante emergencias médicas tomando en cuenta las causas más frecuentes de atención en los servicios de emergencia de medicina a nivel nacional, en los años de realización del piloto, así como estudios previos en una población similar ${ }^{(24)}$. Los ítems evaluados fueron: la respuesta ante emergencias (atragantamiento, quemadura, convulsión, descarga eléctrica, hemorragia activa, atropello o intoxicación por sustancia tóxica); sospecha de emergencias (infarto cardiaco y fractura) y conocimiento del número local de emergencias. Para su confección se contó con la colaboración de tres profesionales en emergencias y desastres, cada uno con más de 30 años de experiencia en el tema.

Se evaluó la validez de constructo a través de juicio de expertos, el cuestionario fue evaluado en la forma y fondo por diez médicos de un hospital público de referencia nacional de la provincia del Callao. Dicha evaluación se realizó con una escala del 0-10, las preguntas que obtuvieron un promedio mayor de ocho fueron consideradas en el cuestionario final, además se realizó los ajustes adecuados a las preguntas que no estaban claramente explicadas. Luego, entre los años 2006-2007, se realizó un piloto, en la primera fase se aplicó el cuestionario a los representantes de doce sociedades científicas de estudiantes de medicina del norte, centro y sur del país. En la última fase del piloto se envió el cuestionario a los representantes estudiantiles de seis países de Latinoamérica (Bolivia, Chile, El Salvador, México, Paraguay y Panamá), que evaluaron la comprensión y replicabilidad de las preguntas en sus respectivas sedes, las pruebas estadísticas mostraron que no hubo diferencias significativas en los promedios de las respuestas que se obtuvieron en las dos fases del piloto.

El cuestionario aplicado estuvo conformado por dos secciones, la socio-educativa (género, edad, año de estudios, universidad de procedencia, participación en 
curso relacionado con emergencias, lugar y momento de dicha participación) y diez preguntas de nivel de conocimiento sobre emergencias médicas, estructuradas de opción múltiple. Para medir el nivel de conocimiento se consideró la escala 0 al 10 , considerando como nota aprobatoria a los encuestados que obtuvieron seis o más preguntas bien respondidas.

\section{PROCEDIMIENTOS Y ÉTICA EN LA INVESTIGACIÓN}

Se convocó a los representantes locales a través de contactos logrados en la Sociedad Científica Médico Estudiantil Peruana (SOCIMEP), se obtuvo la participación de 15 colaboradores para la recolección de datos, el cuestionario fue socializado entre cada delegado y una pregunta fue adaptada a la realidad de cada sede, la que hacía referencia al número telefónico del sistema local de emergencias médicas. Se instruyó a cada delegado para la aplicación del cuestionario en los horarios libres de las actividades académicas y para la respuesta a las dudas de los participantes. La encuesta fue autoaplicada y anónima, los encuestados brindaron su consentimiento de manera verbal antes de resolverla, luego de la explicación del objetivo de la investigación que le hicieran los delegados de sedes.

Análisis de datos. La base se creó en la sede directriz (Universidad Ricardo Palma, Lima), con el programa Excel $® 2007$. Los datos fueron procesados en el paquete estadístico STATA 11.0. En el análisis descriptivo las variables numéricas se analizaron a través de promedios y desviación estándar, evaluándose los supuestos de normalidad y las variables categóricas utilizando frecuencias y porcentajes. Para la estadística analítica se consideró un valor de $p<0,05$ como significativo, se cruzó las variables categóricas utilizando la prueba del

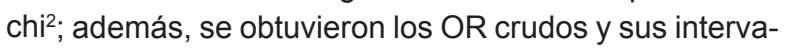
los de confianza al 95\% (IC95\%). El análisis multivariado se hizo con la prueba estadística generalizad linear model, usando la familia gaussian y link logit, se usaron modelos anidados y robustos para disminuir el error por muestras grandes, se obtuvieron los OR ajustados y sus IC95\%.

\section{RESULTADOS}

Participaron 2109 estudiantes de medicina, ninguna de las sedes tuvo una tasa de rechazo mayor al 5\% (rango 1-5\%). La edad promedio fue 21 años (rango 15-32), $1079(51,2 \%)$ fueron de género masculino, el tercer año concentraba mayor cantidad de estudiantes, 458 $(21,7 \%)$ y el quinto año fue el que tuvo menor participación, $292(13,9 \%)$. Del total de encuestados, $1108(52,5 \%)$ Ilevaron un curso relacionado con la enseñanza de las emergencias médicas y, de estos, $708(78,5 \%)$ lo recibieron en la universidad de procedencia; 405 (44,7\%) lo llevaron en los últimos 12 meses. Las características socioeducativas de los participantes se presentan en la Tabla 1.

Tabla 1. Características socioeducativas de estudiantes de medicina de once universidades del Perú.

\begin{tabular}{|c|c|c|}
\hline Características & $\mathbf{n}$ & $(\%)$ \\
\hline \multicolumn{3}{|l|}{ Sexo } \\
\hline Masculino & 1079 & 51,2 \\
\hline Femenino & 1030 & 48,8 \\
\hline Edad (promedio rango) & 20,86 & $15-32$ \\
\hline \multicolumn{3}{|l|}{ Universidad de procedencia } \\
\hline Universidad Nacional - Arequipa & 122 & 5,8 \\
\hline Universidad Nacional de Cajamarca & 119 & 5,6 \\
\hline Universidad Nacional - Chiclayo & 148 & 7,0 \\
\hline Universidad Nacional - Cusco & 148 & 7,0 \\
\hline Universidad Nacional - Huancayo & 119 & 5,6 \\
\hline Universidad Nacional de Piura & 132 & 6,3 \\
\hline Universidad Nacional - Puno & 175 & 8,3 \\
\hline Universidad Nacional - Tacna & 128 & 6,1 \\
\hline Universidad Particular-Arequipa & 195 & 9,2 \\
\hline Universidad Particular-Chimbote & 227 & 10,8 \\
\hline Universidad Particular-Lima & 596 & 28,3 \\
\hline \multicolumn{3}{|l|}{ Año de estudios } \\
\hline Primer año & 458 & 21,7 \\
\hline Segundo año & 493 & 23,4 \\
\hline Tercer año & 547 & 25,9 \\
\hline Cuarto año & 319 & 15,1 \\
\hline Quinto año & 292 & 13,9 \\
\hline \multicolumn{3}{|l|}{ Recibió curso previo del tema } \\
\hline No & 1108 & 52,5 \\
\hline Sí & 1001 & 47,5 \\
\hline \multicolumn{3}{|l|}{ En donde lo recibió } \\
\hline Mi Universidad & 708 & 78,5 \\
\hline Otra Universidad & 51 & 5,7 \\
\hline Institución de salud & 101 & 11,2 \\
\hline Otras & 42 & 4,6 \\
\hline \multicolumn{3}{|l|}{ Cuando lo recibió } \\
\hline No más de 12 meses & 405 & 44,7 \\
\hline 12 a 36 meses & 389 & 42,9 \\
\hline 36 meses o más & 112 & 12,4 \\
\hline \multicolumn{3}{|c|}{ Aprobación según universidad de procedencia } \\
\hline $\begin{array}{l}\text { Universidad Nacional de San Agustín - Are- } \\
\text { quipa }\end{array}$ & 72 & 59,0 \\
\hline $\begin{array}{l}\text { Universidad Nacional de Cajamarca - Caja- } \\
\text { marca }\end{array}$ & 12 & 10,1 \\
\hline $\begin{array}{l}\text { Universidad Nacional Pedro Ruiz Gallo - } \\
\text { Chiclayo }\end{array}$ & 89 & 60,1 \\
\hline $\begin{array}{l}\text { Universidad Nacional de San Antonio Abad } \\
\text { - Cusco }\end{array}$ & 113 & 76,4 \\
\hline $\begin{array}{l}\text { Universidad Nacional del Centro del Perú - } \\
\text { Huancayo }\end{array}$ & 39 & 32,8 \\
\hline Universidad Nacional de Piura - Piura & 52 & 39,4 \\
\hline Universidad Nacional del Altiplano - Puno & 92 & 52,6 \\
\hline $\begin{array}{l}\text { Universidad Nacional Jorge Basadre Groh- } \\
\text { mann- Tacna }\end{array}$ & 68 & 53,1 \\
\hline $\begin{array}{l}\text { Universidad Católica de Santa María-Are- } \\
\text { quipa }\end{array}$ & 61 & 31,3 \\
\hline Universidad Privada San Pedro - Chimbote & 38 & 16,7 \\
\hline Universidad Ricardo Palma - Lima & 200 & 33,6 \\
\hline
\end{tabular}


Tabla 2. Frecuencia de respuestas por cada pregunta del cuestionario aplicado.

\begin{tabular}{|c|c|c|}
\hline & $\mathbf{n}$ & $(\%)$ \\
\hline \multicolumn{3}{|l|}{ Primera acción ante una persona que sufre un atragantamiento y se está ahogando } \\
\hline Realizo la maniobra de Hemlich golpeándole el pecho & 1125 & 53,4 \\
\hline Inclino a la persona hacia delante y le digo que intente toser & 435 & 20,6 \\
\hline Lo golpeo en la zona cervical & 449 & 21,3 \\
\hline Le doy agua en pequeñas cantidades & 60 & 2,8 \\
\hline Lo recuesto sobre el piso e intento darle aire & 40 & 1,9 \\
\hline \multicolumn{3}{|c|}{ Si una persona presenta dolor toráxico y se sospecha de un infarto cardiaco, ¿cuál sería su primera acción? } \\
\hline Inicio reanimación cardio-pulmonar y si no responde doy respiración boca a boca & 394 & 18,7 \\
\hline Llamo a las personas que tenga alrededor para que me ayuden a movilizarlo & 147 & 7,0 \\
\hline Le doy un analgésico u otro fármaco que le disminuya el dolor & 58 & 2,7 \\
\hline Lo echo y le empiezo a realizar el masaje cardíaco & 504 & 23,9 \\
\hline $\begin{array}{l}\text { Sentarlo, desajustarle todo lo que le cause presión (p. ejem.: cinturón, cuello de camisa, etc.) y } \\
\text { Ilamar a emergencias }\end{array}$ & 1006 & 47,7 \\
\hline \multicolumn{3}{|l|}{ Una persona que cae, está consciente y se sospecha de una fractura } \\
\hline Le doy un medicamento que calme su dolor hasta que llegue la ayuda & 87 & 4,1 \\
\hline Trato de inmovilizar la zona para que no se haga más daño & 1745 & 82,7 \\
\hline Le aplico una crema analgésica a la zona afectada & 70 & 3,3 \\
\hline Lo levanto lo antes posible para que no siga en la zona que ocurrió el incidente & 121 & 5,8 \\
\hline Le cubro la zona afectada para que no se lastime más & 86 & 4,1 \\
\hline \multicolumn{3}{|l|}{ Si una persona sufre quemadura por líquido caliente } \\
\hline Le echo querosene para evitar que se expanda la quemadura & 36 & 1,7 \\
\hline Le descubro la zona para que se refresque & 345 & 16,4 \\
\hline Le hecho alcohol o agua oxigenada según lo que tenga a la mano & 89 & 4,2 \\
\hline Lo expongo al chorro de agua por unos 15 a 20 minutos & 1554 & 73,7 \\
\hline Uso crema dentífrica para aliviar el dolor y que no deje marca & 85 & 4,0 \\
\hline \multicolumn{3}{|l|}{ Si una persona está convulsionando en el suelo } \\
\hline Le introduzco los dedos a la boca para que no se trague su lengua & 152 & 7,2 \\
\hline Trato de sujetarlo para evitar que siga convulsionando & 121 & 5,7 \\
\hline Le introduzco un objeto a la boca para que no se muerda su lengua y se la trague & 1016 & 48,2 \\
\hline Le doy gaseosa, café o un líquido caliente para que se recupere & 66 & 3,1 \\
\hline Tratar de acomodarle y proteger la cabeza para que no se lastime o siga lastimando & 754 & 35,8 \\
\hline \multicolumn{3}{|l|}{ Si una persona ha recibido una descarga eléctrica, ¿qué acción primaria tomaría? } \\
\hline Trato de moverlo para ver su estado de consciencia & 168 & 8,0 \\
\hline Mido de inmediato sus funciones vitales & 893 & 42,3 \\
\hline Le doy medicación para que no entre en paro cardiaco & 63 & 3,0 \\
\hline Compruebo si aún está recibiendo la descarga y trato de impedirlo & 770 & 36,5 \\
\hline Si no lo veo moverse inicio reanimación cardio-pulmonar & 215 & 10,2 \\
\hline \multicolumn{3}{|l|}{ Si veo que la persona tiene hemorragia severa activa, ¿qué acción primaria tomaría? } \\
\hline Hago presión directa en la zona y la elevo & 753 & 35,7 \\
\hline Vendarle la zona y cambiar las vendas cada vez que se empapen & 150 & 7,1 \\
\hline Realizo un torniquete en la zona afectada para evitar la hemorragia & 1112 & 52,7 \\
\hline Le aplico alcohol de inmediato para evitar que se infecte & 65 & 3,1 \\
\hline Si tengo querosene a la mano se lo aplico para controlar la hemorragia & 29 & 1,4 \\
\hline \multicolumn{3}{|l|}{ ¿Cuál es el número de teléfono para emergencias médicas? } \\
\hline 911 & 659 & 31,3 \\
\hline 116 & 577 & 27,4 \\
\hline 103 & 115 & 5,4 \\
\hline 105 & 530 & 25,1 \\
\hline 115 & 228 & 10,8 \\
\hline \multicolumn{3}{|l|}{ Una persona que ha sido atropellada y está inconsciente, ¿cuál sería su primera acción? } \\
\hline Lo movilizo y le inicio la reanimación cardio-pulmonar & 219 & 10,4 \\
\hline Lo saco de inmediato de la zona & 233 & 11,1 \\
\hline No lo muevo y aseguro su vía respiratoria & 1572 & 74,5 \\
\hline Tiro de uno de sus dedos para ver su reacción & 57 & 2,7 \\
\hline Jalo una de sus piernas y veo su estado de consciencia & 28 & 1,3 \\
\hline \multicolumn{3}{|l|}{ ¿Qué haría si una persona ha ingerido sustancia tóxica por vía oral? } \\
\hline Darle leche para desintoxicarlo & 237 & 11,2 \\
\hline Introducirle el dedo a la boca para provocarle el vómito & 447 & 21,2 \\
\hline Darle aceite para provocarle el vómito & 96 & 4,6 \\
\hline Darle agua en pequeñas cantidades para que pase la sustancia & 103 & 4,9 \\
\hline Evacuarlo al hospital más cercano, si es posible con una muestra de lo ingerido & 1226 & 58,1 \\
\hline
\end{tabular}


La nota promedio que obtuvieron de las preguntas sobre emergencias médicas establecidas en el cuestionario fue 4,95, con una desviación estándar de 1,76; 13 estudiantes sacaron 0 de nota y uno sacó 10 puntos. El $25 \%$ obtuvo $\leq 4$ puntos, el $50 \%$ obtuvo $\leq 5$ puntos y el $75 \%$ obtuvo $\leq 6$ puntos. Consiguieron de 8-10 puntos $123(5,8 \%)$ estudiantes. Las preguntas que mejor fueron respondidas fueron las que indagaron la mejor manera de atender a un paciente con sospecha de fractura, $82,7 \%$ respondió que inmovilizarían el miembro; cómo atender a una persona atropellada, 74,5\% no lo movería de la escena y aseguraría su vía aérea; mientras que cómo actuarían ante un quemado por elemento caliente, $73,7 \%$ lo expondría a un chorro de agua. Las preguntas que peores respuestas tuvieron fueron aquellas relacionadas con el actuar ante una persona que se ahoga por atragantamiento alimenticio, el $53,4 \%$ le realizaría una maniobra de Hemlich golpeándole el pecho; en tanto sobre la pregunta de qué acción tomaría ante una hemorragia grave activa, el $52,7 \%$ le haría un torniquete; en el modo de actuar ante una persona que convulsiona, el 48,2\% le colocaría un objeto en la boca para que no se muerda la lengua. Las frecuencias de respuesta por cada pregunta se muestran en la Tabla 2.

En el análisis bivariado se encontró una fuerte asociación entre los que tuvieron nota aprobatoria ( $\geq 6$ puntos) y el grupo etario $(p<0,001)$, la universidad de procedencia $(p<0,001)$, la etapa académica $(p<0,001)$, haber recibido un curso de emergencias médicas $(p<0,001)$, cuando recibieron dicho curso $(p<0,001)$ y dónde lo recibieron $(p<0,001)$ Tabla 3.

En el análisis multivariado, se mantuvo asociado el haber tenido promedio aprobatorio con la universidad de procedencia ( $p<0,001$, OR: 0,45, IC95\% 0,38-0,54), la etapa académica ( $p<0,001$, OR: 1,55, IC95\% 1,28-1,87), cuándo recibieron un curso de emergencias médicas $(p<0,001$, OR: 0,62 , IC95\% 0,50-0,77) y el género ( $p=0,001$, OR: 1,38, IC95\% 1,15-1,65) (Tabla 4).

\section{DISCUSIÓN}

Nuestro objetivo fue evaluar el nivel de conocimientos sobre emergencias médicas de los estudiantes de medicina de once universidades peruanas. Basándonos en los resultados obtenidos, se encontró que $60,4 \%$ de los estudiantes encuestados tuvieron resultados desaprobatorios, el promedio fue de 4,95 sobre un máximo de 10. Se encontraron además diferencias significativas entre las variables socio-educativas de los que aprobaron y no aprobaron el cuestionario. De estas, las variables no intervenibles fueron la universidad de procedencia, la etapa académica y el género, siendo la única variable modificable cuándo recibió un curso sobre el tema.

Aquellos que recibieron un curso relacionado con el tema el último año, tuvieron $38 \%$ menos oportunidad de desaprobar el cuestionario (OR ajustado: 0,62; IC95\%: $0,50-0,77)$. En una población similar se observó una mejora del nivel de conocimiento del $41 \%$ luego de una intervención educativa ${ }^{(25)}$. En poblaciones de Europa, Asia y Oceanía se muestran resultados similares luego de intervenciones educativas ${ }^{(26-28)}$. Los datos sugieren la gran influencia que tiene la práctica como herramienta para el aprendizaje, sobre todo en temas donde la obtención de la pericia se basa en la experiencia, así como en la mejora diagnóstica y el reconocimiento de situaciones que requieren la intervención médica inmediata ${ }^{(29,30)}$.

Tabla 3. Análisis bivariado del nivel de conocimientos de estudiantes de medicina sobre emergencias médicas.

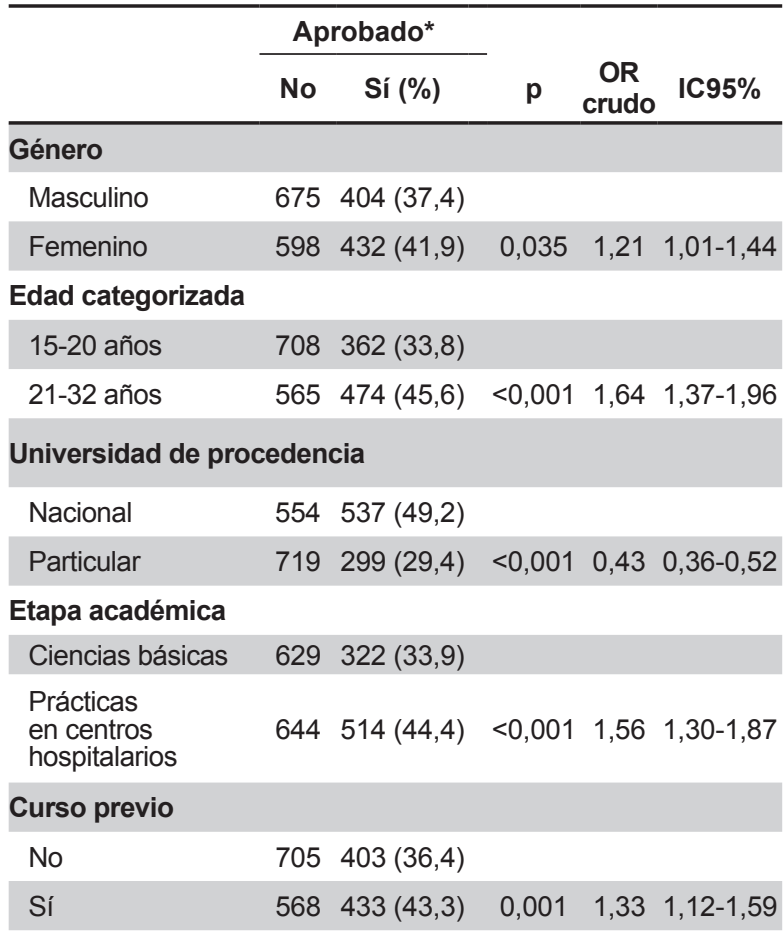

\section{Cuándo recibió curso del tema}

$\begin{array}{lrllll}\text { El mismo año } & 203202(49,9) & \\ \begin{array}{l}\text { No recibió ese } \\ \text { año curso del tema }\end{array} 1070634(37,2) & <0,001 \quad 0,60 \quad 0,48-0,75\end{array}$

\section{Donde recibió curso del tema}

\begin{tabular}{|c|c|c|c|}
\hline Universidad & 416 & $343(45,2)$ & \\
\hline $\begin{array}{l}\text { No recibió curso } \\
\text { del tema en alguna } \\
\text { universidad }\end{array}$ & 857 & $\begin{array}{c}493 \\
(36,5)\end{array}$ & $<0,001 \quad 0,70 \quad 0,58-0,84$ \\
\hline
\end{tabular}

* Se consideró aprobado aquellos participantes que repondieron correctamente a seis o más preguntas del cuestionario de diez items. 
Tabla 4. Análisis multivariado: factores asociados a aprobar el cuestionario sobre nivel de conocimientos de estudiantes de medicina sobre emergencias médicas.

\begin{tabular}{lccc}
\hline & $\mathrm{p}$ & $\begin{array}{c}\text { OR } \\
\text { ajustado }\end{array}$ & $\mathrm{IC95 \%}$ \\
\hline $\begin{array}{l}\text { Universidad de } \\
\text { procedencia } \\
\text { (particular vs. nacional) }\end{array}$ & $<0,001$ & 0,45 & $0,38-0,54$ \\
$\begin{array}{l}\text { Etapa académica } \\
\text { (prácticas hospitalarias vs. } \\
\text { ciencias básicas) }\end{array}$ & $<0,001$ & 1,55 & $1,28-1,87$ \\
$\begin{array}{l}\text { Recibió curso } \\
\text { del tema (no vs. si) }\end{array}$ & $<0,001$ & 0,62 & $0,50-0,77$ \\
$\begin{array}{l}\text { Género } \\
\text { (femenino vs. masculino) }\end{array}$ & 0,001 & 1,38 & $1,15-1,65$ \\
\hline
\end{tabular}

Asimismo, aquellos que cursaban prácticas en centros hospitalarios tuvieron $55 \%$ más oportunidad de aprobar el cuestionario (OR ajustado: 1,55; IC95\%: 1,28-1,87). En el tema de emergencias médicas, existen reportes de mejora en el nivel de conocimientos conforme los estudiantes de poblaciones similares avanzan hacia años superiores ${ }^{(31-33)}$. Lo que apunta a que la etapa académica sigue siendo un factor fundamental para la preparación de los futuros médicos, puesto que el paso de las aulas a los hospitales es la base de la relación médico-paciente y del aprendizaje mediante la práctica.

No hemos encontrado reportes en poblaciones similares que corroboren que el provenir de alguna universidad nacional o ser del género femenino sean factores asociados con la mejor respuesta ante emergencias médicas, esto debe ser corroborado con estudios que tengan una mejor representatividad de las universidades nacionales y particulares de todas las regiones del país. Los resultados de las preguntas que más y menos acertaron nos muestran dos posiciones opuestas, por un lado, tienen buena noción de la atención primaria ante ciertas situaciones (fractura, atropello y quemadura), por el contrario, aún existen tópicos de emergencia médica que se ven influenciados por el pensamiento del ciudadano común como por ejemplo el uso del torniquete como medida de control de la hemorragia o el colocar un objeto en la boca de un paciente que convulsiona para evitar que se muerda la lengua.

Durante el estudio tuvimos algunas limitaciones: el muestreo no fue probabilístico debido a la política de reserva de datos de los alumnos, sobre todo de las universidades particulares. A pesar de esta limitación, se tuvo cuidado en que las universidades participantes sean adecuadamente representadas, según la proporción de estudiantes matriculados en el periodo de realización del estudio, consideramos que no hubieron diferencias entre la población y la muestra, y si es que las hubiese, serían mínimas y no diferenciales; por motivos de contactos y permisos no se incluyó alguna de las dos universidades públicas que existen en Lima, solo fue incluida una universidad privada, cuya población está claramente sobrerrepresentada en la encuesta (casi $30 \%$ del total), esto genera un sesgo en la representatividad del estudio con respecto al universo de estudiantes peruanos, pero no lo invalida. Los intervalos de confianza no son la medida más confiable por ser un muestreo no probabilístico, sin embargo, al tener una muestra por encima de nuestro mínimo requerido, obtenerla con un error de 0,02 y al ser un estudio multicéntrico los resultados se pueden considerar como un proxy de la real situación en la población evaluada. Por último, no se midieron otras variables que son influyentes en el nivel de conocimiento de los estudiantes, solo unas cuantas variables socio-educativas generales, por lo que futuros estudios deberían tratar de abordar este tema desde diversas perspectivas educativas y con mayor cantidad de factores influyentes.

Los datos presentados son los resultados de la mayor muestra estudiantil evaluada en nuestro país, en un aspecto académico específico. Dichos resultados sirven para explorar algunos aspectos influyentes en la formación del que será el personal de salud peruano en algunos años. Las diferencias encontradas en las variables analizadas sugieren que, como era de esperar, el nivel de conocimiento sobre emergencias médicas depende de los cursos de actualización y complementación que tenga el estudiante de medicina en su record académico, así como la formación práctica de campo que le brinde su casa de estudios. Fue inesperado encontrar diferencias en el nivel de conocimiento según el género y la procedencia de la casa de estudios, obteniendo promedios desaprobatorios los hombres y quienes estudiaban en una universidad privada.

A pesar de que los resultados obtenidos no permiten inferencias concluyentes, estos exploran características estudiantiles relevantes que influyen en la obtención de conocimientos. No solo en el tema de emergencias médicas, sino también aquellas ramas de la medicina que se basan en la práctica de campo como mecanismo de aprendizaje. Las instituciones responsables deben de velar por la adecuada enseñanza de los que un futuro serán los recursos humanos del sector salud. Esto debería ser explorado con más detalle por futuros investigadores y grupos afines con la investigación en los recursos humanos.

Se concluye que el nivel de conocimiento sobre emergencias médicas de los estudiantes de las once universidades evaluadas no es bueno. 


\section{AGRADECIMIENTOS}

A los profesionales de la salud que nos apoyaron en la construcción del instrumento y en los pasos posteriores, sobre todo al Dr. Alfredo Huerta García y a Luis Donayre Reyes. Así como al equipo encargado del trabajo de campo que contribuyó en diferentes etapas del estudio y a la Sociedad Científica de Estudiantes de Medicina de la Universidad Ricardo Palma.

\section{Contribuciones de Autoría}

CRM participó en la concepción y diseño del trabajo, análisis e interpretación de datos y redacción de la primera versión del manuscrito. CCQO participó en el análisis e interpretación de datos y redacción de la primera versión del manuscrito, CMV participó en la concepción y diseño del trabajo, interpretación de datos y redacción de la primera versión del manuscrito. KQP participó en la concepción y diseño del trabajo, interpretación de datos y redacción de la primera versión del manuscrito, CAO participó en la concepción y diseño del trabajo, interpretación de datos y redacción de la primera versión del manuscrito. Todos los autores revisaron en forma crítica versiones preliminares del manuscrito y aprobaron la versión final del trabajo.

\section{Fuentes de Financiamiento \\ Autofinanciado.}

\section{Conflictos de Interés}

Los autores no declaran conflictos de interés.

\section{REFERENCIAS BIBLIOGRÁFICAS}

1. Organización Mundial de la Salud. El Botiquín médico interinstitucional de emergencia. Geneva: OMS; 2006. Disponilbe en: http://www.who.int/medicines/publications/ WEB_IEHK_SP.pdf.

2. Enami M, Takei $\mathbf{Y}$, Goto $\mathbf{Y}$, Ohta $\mathbf{K}$, Inaba $\mathbf{H}$. The effects of the new CPR guideline on attitude toward basic life support in Japan. Resuscitation. 2010;81(5):562-7.

3. Morales-Soto N. Emergencias y Desastres. Revista Peruana de Medicina Experimental y Salud Pública. 2008;25(1):2.

4. Ministerio de Salud del Perú (MINSA). Accidentes de tránsito una epidemia mundial. Boletín estadístico de salud. Lima MINSA; 2009.

5. Morales-Soto N. Algunas consideraciones para la organización de servicios de salud para emergencias y desastres. An Fac med. 2001;62(1):44-55.

6. Naciones Unidas en el Perú. Desastres y crisis humanitarias [Internet]. Lima: ONU en el Perú; 2004 [citado el 14 de marzo del 2011]. Disponible en: http://www.onu.org.pe/ Publico/infocus/accionhumanitaria.aspx.
7. Allison KP, Kilner T, Porter KM, Thurgood A. Pre-hospital care--the evolution of a course for undergraduates. Resuscitation. 2002;52(2):187-91.

8. Goulon M. [Teaching emergency medicine]. Bull Acad Nat Med. 1991 Mar;175(3):403-17.

9. Seefeld AW. Lessons learned from working in emergency departments in Cape Town, South Africa : a final-year medical student's perspective. S Afr Med J. 2007;97(2):78.

10. Creutzfeldt J, Hedman L, Medin C, Wallin CJ, Hendrick A, Youngblood P, et al. Implementing virtual worlds for systematic training of prehospital CPR in medical school. Stud Health Technol Inform. 2007;125:82-4.

11. Harrison GA, Hillman KM, Fulde GW, Jacques TC. The need for undergraduate education in critical care. (Results of a questionnaire to year 6 medical undergraduates, University of New South Wales and recommendations on a curriculum in critical care). Anaesth Intensive Care. 1999;27(1):53-8.

12. Ruppert M, Reith MW, Widmann JH, Lackner CK, Kerkmann R, Schweiberer L, et al. Checking for breathing: evaluation of the diagnostic capability of emergency medical services personnel, physicians, medical students, and medical laypersons. Ann Emerg Med. 1999;34(6):720-9.

13. Perry Z, Busiba Z, Uziel E, Meiri G. [Crisis intervention-the summary of a unique interventional program for medical students]. Harefuah. 2009;148(2):87-92, 139-40.

14. Saunders NA, Wallis BJ. Learning decision-making in clinical medicine: a card game dealing with acute emergencies for undergraduate use. Med Educ. 1981;15(5):323-7.

15. Weller JM. Simulation in undergraduate medical education: bridging the gap between theory and practice. Med Educ. 2004;38(1):32-8.

16. Cassoobhoy M, Wetterhall SF, Collins DF, Cantey PT, Iverson CJ, Rudnick JR, et al. Development of an interactive bioterrorism and emerging infections curriculum for medical students and internal medicine residents. Public Health Rep. 2005;120 Suppl 1:59-63.

17. Hunskaar S, Seim SH. Medical students' experiences in medical emergency procedures upon qualification. Med Educ. 1985;19(4):294-8.

18. Luscher F, Hunziker S, Gaillard V, Tschan F, Semmer NK, Hunziker PR, et al. Proficiency in cardiopulmonary resuscitation of medical students at graduation: a simulatorbased comparison with general practitioners. Swiss Med Wkly. 2010;140(3-4):57-61.

19. Fitch MT. Using high-fidelity emergency simulation with large groups of preclinical medical students in a basic science course. Med Teach. 2007;29(2-3):261-3

20. Dirks B, Keller AW, Jager G, Ochsner W, Weisser FO, Georgieff M. [The Ulm emergency medicine training course. 1: Concept]. Anasthesiol Intensivmed Notfallmed Schmerzther. 1996;31(3):168-71.

21. Tipa RO, Bobirnac G. Importance of basic life support training for first and second year medical students--a personal statement. J Med Life. 2010;3(4):465-7.

22. Walcher F, Russeler M, Nurnberger F, Byhahn C, Stier $\mathbf{M}$, Mrosek $\mathbf{J}$, et al. [Mandatory elective course in emergency medicine with instructions by paramedics improves practical training in undergraduate medical education]. Unfallchirurg. 2010;114(4):340-4. 
23. Wall D, Bolshaw A, Carolan J. From undergraduate medical education to pre-registration house officer year: how prepared are students? Med Teach. 2006;28(5):435-9.

24. Montes Arias LL. Evaluación teórica de los conocimientos y habilidades sobre primeros auxilios en estudiantes de medicina de la UNSA. Arequipa: Universidad Nacional de San Agustín; 1996.

25. Nuñez-Zevallos GE. Influencia de la educación en los conocimientos y habilidades sobre primeros auxilios en estudiantes de educación inicial de la Universidad Nacional de San Agustín de Arequipa 1996. Arequipa: Universidad Nacional de San Agustín; 1996.

26. Khan A, Shaikh S, Shuaib F, Sattar A, Samani SA, Shabbir $\mathbf{Q}$, et al. Knowledge attitude and practices of undergraduate students regarding first aid measures. J Pak Med Assoc. 2010;60(1):68-72.

27. Polglase RF, Parish DC, Buckley RL, Smith RW, Joiner TA. Problem-based ACLS instruction: a model approach for undergraduate emergency medical education. Ann Emerg Med. 1989 Sep;18(9):997-1000.

28. Kelly AM, Ardagh MW. Does learning emergency medicine equip medical students for ward emergencies? Med Educ. 1994 Nov;28(6):524-7.

29. Kliegel A, Scheinecker W, Sterz F, Eisenburger P, Holzer M, Laggner AN. The attitudes of cardiac arrest survivors and their family members towards CPR courses. Resuscitation. 2000;47(2):147-54
30. Chapman JJ, Weiss SJ, Haynes ML, Ernst AA. Impact of EMS education on emergency medicine ability and career choices of medical students. Prehosp Emerg Care. 1999 Apr-Jun;3(2):163-6.

31. Celenza A, Jelinek GA, Jacobs I, Kruk C, Graydon R, Murray L. Implementation and evaluation of an undergraduate emergency medicine curriculum. Emerg Med (Fremantle). 2001;13(1):98-103.

32. Weller J, Robinson B, Larsen P, Caldwell C. Simulationbased training to improve acute care skills in medical undergraduates. N Z Med J. 2004;117(1204):U1119.

33. Reta de Rosas AM, López MJ, Montbrun M, Ortiz A, Lía Vargas A. Competencias médicas y su evaluación al egreso de la carrera de medicina en la Universidad Nacional de Cuyo (Argentina). Educación Médica. 2006;9:75-83.

Correspondencia: Christian R. Mejia.

Dirección: Av. Las Palmeras Mz N1 Lt 33, Villa del Norte-Los

Olivos, Lima 33, Perú.

Teléfono: (511) 997-643516

Correo electrónico: chrimeal@hotmail.com

\section{Consulte las ediciones anteriores de la} Revista Peruana de Medicina Experimental y Salud Pública en

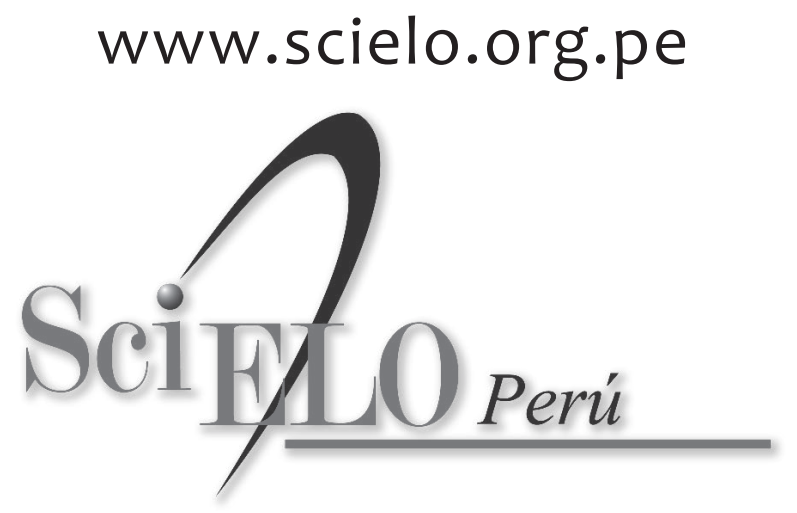

